\title{
On Quasi-Normal and Quasi-Regular Spaces in Hereditary Generalized Topological Spaces
}

\author{
M.Vigneshwaran ${ }^{1}$, K. Baby ${ }^{2}$ \\ ${ }^{1}$ Assistant Professor, Department of Mathematics, Kongunadu arts and Science College, Coimbatore-29, Tamilnadu, India \\ ${ }^{2}$ Ph.D Research scholar, Department of Mathematics, Kongunadu arts and Science College, Coimbatore-29, Tamilnadu, India.
}

\begin{abstract}
The concept of generalized open sets in generalized topologies was investigated by Csaszar [2]. In this paper we introduce spaces namely Quasi $\mu-\mathcal{H}$-regular spaces, Almost $\mu$ - $\mathcal{H}$-Normal spaces and Quasi ultra $\mu$ - $\mathcal{H}$-Normal spaces with a fixed set of parameters and obtain some properties in the light of these notion. We also introduce Quasi $\mu_{\beta}-\mathcal{H}_{\text {-regular spaces and } Q u a s i} \mu_{\beta}-\mathcal{H}$ normal spaces and investigate some properties of these new notions by using some basic properties of ( $\mu$, $\lambda$ )-continuity in generalized topological spaces introduced by M.Rajamani, V.Inthumathi and R.Ramesh [5]. Moreover we obtain relations between Quasi $\mu$ - $\mathcal{H}$ normal spaces and Almost $\mu$ - $\mathcal{H}$-normal spaces with respect to $(\mu, \lambda)$-continuity and $(\mu, \lambda)$-open map.
\end{abstract}

Keywords: Quasi $\mu$ - $\mathcal{H}$-normal spaces, Quasi $\mu$ - $\mathcal{H}$-regular spaces, Quasi ultra $\mu$ - $\mathcal{H}$-Normal space, Almost $\mu$ - $\mathcal{H}$-Normal spaces, $(\mu, \lambda)$ continuity and $(\mu, \lambda)$-open, $(\mu, \lambda) \beta$-irresolute, $(\mu, \lambda) \beta$-continuous, $(\mu, \lambda)$ R-irresolute, $(\mu, \lambda)$ R-pre-closed.

\section{Introduction and Preliminaries}

The idea of generalized topology and hereditary class was introduced and studied by Csaszar[2]. A subfamily $\mu$ of $\mathrm{P}(X)$ is called a generalized topology if $\phi \in \mu$ and union of elements of $\mu$ belongs to $\mu$. The space $X$ together with the generalized topology $\mu$ is said to be generalized topological space and denoted by $(X, \mu) . i_{\mu}(A)$ and $c_{\mu}(A)$ denotes the interior and closure of $A$ in $(X, \mu)$. The elements of $\mu$ are called open and is denoted by $\mu$-open. The complement of $\mu$ open is $\mu$-closed. We say a hereditary class $\mathcal{H}$ on $(X, \mu)$ is a non-empty collection of subset of $\mathrm{X}$ such that $A \subseteq B, B \in$ $\mathcal{H}$ implies

$A \in \mathcal{H}$. With respect to the generalized topology $\mu$ and a hereditary class $\mathcal{H}$, for a subset $A$ of X we define $A_{\mu}{ }^{*}(\mathcal{H})$ or simply $A_{\mu}{ }^{*}=\{x \in X: M \cap A \notin \mathcal{H}$ for every $M \in \mu$ such that $x \in M\}$. The closure $c_{\mu}{ }^{*}(A)=A \cup A_{\mu}{ }^{*}(\mathcal{H})$. The space $(X, \mu)$ with the hereditary class $\mathcal{H}$ is called hereditary generalized topological space and denoted by $(X$, $\mu, \mathcal{H})$. A subset $A$ of $(X, \mu)$ is $\mu \alpha$-open [2] (resp. $\mu$-semi open [2], $\mu$-pre open [2], $\mu \beta$-open[2]), if $A \subseteq$ $i_{\mu}\left(c_{\mu}\left(i_{\mu}(A)\right)\right)\left(\operatorname{resp} . A \subseteq c_{\mu}\left(i_{\mu}(A)\right), A \subseteq i_{\mu}\left(c_{\mu}(A)\right)\right.$, $A \subseteq c_{\mu}\left(i_{\mu}\left(c_{\mu}(A)\right)\right)$. We denote the family of all $\mu \alpha$-open sets, $\mu$-semi open sets, $\mu$-pre open sets and $\mu \beta$-open sets by $\alpha(\mu), \sigma(\mu), \pi(\mu)$ and $\beta(\mu)$ respectively. On generalized topology, $\mu \subseteq \alpha(\mu) \subseteq \pi(\mu) \subseteq \beta(\mu)$.

In GTS $c_{\mu_{\alpha}}(A)$ and $c_{\mu_{\beta}}(A)$ denotes $\alpha$-closure of $A$ and $\beta$ closure of $A$ in $(X, \mu)$ respectively. A subset $A$ of $(X, \mu)$ is said to be $\mu$ - regular open[4] if $A=i_{\mu}\left(c_{\mu}(A)\right)$ and the complement is $\mu$-regular closed. The finite union of $\mu$ regular open sets is called $\mu \pi$-open sets and its complement is $\mu \pi$-closed set. A set $A$ is said to be $\mu$ g-closed [2] if, $c_{\mu}(A) \subseteq A$ whenever $A \subseteq U$ and $U$ is $\mu$-open and its complement is $\mu$ g-open.

Definition 1.1: For a subset A of hereditary generalized topological space $(X, \mu, \mathcal{H})$

i) $A_{\mu_{\alpha}}{ }^{*}(\mathcal{H})=\{x \in X: M \cap A \notin \mathcal{H}$ for every $M \in \alpha(\mu)$ such that $x \in M\}$ [2].

ii) $A_{\mu_{\beta}}{ }^{*}(\mathcal{H})=\{x \in X: M \cap A \notin \mathcal{H}$ for every $M \in \beta(\mu)$ such that $x \in M\}$ [2].

Definition 1.2: A subset A of a hereditary generalized topological space $(X, \mu, \mathcal{H})$ is said to be $\mu^{*}$-closed [3] if $A_{\mu}{ }^{*}(\mathcal{H}) \subseteq A$ and $\mu_{\beta}{ }^{*}$-closed [3] if $A_{\mu_{\beta}}{ }^{*}(\mathcal{H}) \subseteq A$. Then ${c_{\mu_{\beta}}}^{*}(A)=A \cup A_{\mu_{\beta}}{ }^{*}(\mathcal{H})$.

Definition 1.3: Let $(X, \mu)$ and $(Y, \lambda)$ be generalized topologies. A function $f:(X, \mu) \rightarrow(Y, \lambda)$ is said to be

i) $(\mu, \lambda)$-continuous if for every closed set $V$ in $(Y, \lambda)$ (denoted by $\lambda$-closed set), $f^{-1}(V)$ is $\mu$-closed,

ii) $(\mu, \lambda)$-open if for every $\mu$-open set $U, f(U)$ is open in $(Y, \lambda)$ (denoted by $\lambda$-open). 


\section{International Journal of Science and Research (IJSR) \\ ISSN (Online): 2319-7064 \\ Index Copernicus Value (2013): 6.14 | Impact Factor (2015): 6.391}

\section{Quasi $\mu-\mathcal{H}$-regular spaces, Almost $\mu-\mathcal{H}$ - Normal spaces and Quasi ultra $\mu$ - $\mathcal{H}$-Normal space}

Definition 2.1: A hereditary generalized topological space $(X, \mu, \mathcal{H})$ is said to be Quasi $\mu-\mathcal{H}$-regular space if for every $\mu \pi$-closed set $A$ and a point $X \notin A$, there exist $\mu$-open sets $U$ and $V$ such that $A-U \in \mathcal{H}, x \in V$ and $U \cap V \in \mathcal{H}$.

Definition 2.2: Let $(X, \mu, \mathcal{H})$ be a hereditary generalized topological spaces. A space $(X, \mu, \mathcal{H})$ is said to be quasi $\mu$ $\mathcal{H}$ - normal if for every pair of $\mu \pi$-closed sets $A$ and $B$ of $X$, there exist $\mu$-open sets $U$ and $V$ such that $A-U \in \mathcal{H}$, $B-V \in \mathcal{H}$, and $U \cap V \in \mathcal{H}$.

Theorem 2.3: Let $(X, \mu, \mathcal{H})$ be a Hereditary generalized topological space.

Then the followings are equivalent:

(a) $\mathrm{X}$ is a quasi $\mu-\mathcal{H}$-regular space.

(b) for each point $x \in X$ and for each $\mu \pi$-open neighbourhood $F$ of $x$, there exists a $\mu$-open set $V$ of $X$ such that $c_{\mu}{ }^{*}(V)-F \in \mathfrak{H}$.

(c) For each point $x \in X$ and for each $\mu \pi$-closed set $A$ not containing, there exists a $\mu$-open set $V$ of $X$ such that $c_{\mu}^{*}(V) \cap A \in \mathcal{H}$.

\section{Proof:}

$(a) \Rightarrow(b)$ Let $F$ be $\mu \pi$-open neighbourhood of $x$. Then there exist a $\mu \pi$-open subset $G$ of $X$ such that $x \in G \subseteq F$. Since $G^{c}$ is $\mu \pi$-closed and $x \in G$ by hypothesis, there exist disjoint $\mu$-open sets $U$ and $V$ such that $G^{\mathcal{c}}-U \in \mathcal{H}, x \in V$ and $U \cap V \in \mathcal{H}$ and so $V-$ $U^{c} \in \mathcal{H}$. Since $U^{c}$ is $\mu$-closed, $c_{\mu}{ }^{*}(V)-U^{c} \in \mathcal{H}$ implies $U^{c}-G \in \mathcal{H}$. Hence $c_{\mu}{ }^{*}(V)-F \in \mathcal{H}$.

(b) $\Rightarrow(a)$ : Let $F^{c}$ be any $\mu \pi$-closed set and $x \notin F^{c}$. Then $x \in F$ and $F$ is $\mu \pi$-open

neighbourhood of $x$. By hypothesis, there exist a $\mu$-open set $V$ of $x$ such that $x \in V$ and $c_{\mu}{ }^{*}(V)-F \in \mathcal{H}$, which implies $F^{c}-c_{\mu}{ }^{*}(V) \in \mathcal{H}$. Then $\left(c_{\mu}{ }^{*}(V)\right)^{c}$ is $\mu$ open set containing $F^{c}$ and $V \cap\left(c_{\mu}{ }^{*}(V)\right)^{c} \in \mathcal{H}$. Therefore is $X$ quasi $\mu-\mathcal{H}$-regular space.

(b) $\Rightarrow(c)$ : Let $x \in X$ and $A$ be $\mu \pi$-closed set such that $x$ $\notin A$. Since $A^{c}$ is $\mu \pi$-open neighbourhood of $x$ and by hypothesis, there exist a $\mu$-open set $V$ of $X$ such that $c_{\mu}{ }^{*}(V)-A^{\mathcal{c}} \in \mathcal{H}$ and $c_{\mu}{ }^{*}(V) \cap A \in \mathcal{H}$.

(c) $\Rightarrow(a)$ set $x \in X$ and $A$ be a $\mu \pi$-closed set such that $x \notin A$. By hypothesis, there exists $\mu$-open set $U$ such that $c_{\mu}{ }^{*}(V) \cap A \in \mathcal{H}$. Let $V=X-c_{\mu}{ }^{*}(U)$. Since $V$ is $\mu$ open set and $U \cap V \in \mathcal{H}, X$ is quasi $\mu-\mathcal{H}$-regular space.

Definition 2.4: Let $(X, \mu)$ and $(Y, \lambda)$ be generalized topologies. A function $f:(X, \mu) \rightarrow(Y, \lambda)$ is said to be

i) Completely $(\mu, \lambda)$-irresolute if for every $\pi$-closed set $V$ in $(Y, \lambda)$ (denoted by $\lambda \pi$ - closed set), $f^{-1}(V)$ is $\pi$-closed in $(X, \mu)$ (denoted by $\mu \pi$-closed).

ii) Completely $(\mu, \lambda)$-continuous if for every $\lambda$-closed set $V$, $f^{-1}(V)$ is $\mu \pi$-closed.

iii) Almost $(\mu, \lambda)$-open if for every $\pi$-open set $V$ in $(X, \mu)$ (denoted by $\mu \pi$-open set) $f(V)$ is $\pi$-open in $(Y, \lambda)$ (denoted by $\lambda \pi$-open).

iv) Almost $(\mu, \lambda)$-closed if for every $\mu \pi$-closed set $F, f(F)$ is $\lambda \pi$-closed.

v) Perfectly $(\mu, \lambda)$-continuous if for every open set $F$ in $(Y$, $\lambda$ ) (denoted by $\lambda$-open set), $f^{-1}(F)$ is $\mu$-open and $\mu$-closed.

vi) $(\mu, \lambda)$-R-irresolute if for every regular-closed set $V$ in $(Y$, $\lambda$ ) (denoted by $\lambda$-regular-closed), $f^{-1}(V)$ is $\mu$-regular closed.

vii) $(\mu, \lambda) \beta$-irresolute if for every $\beta$-closed set $V$ in $(Y, \lambda)$ (denoted by $\lambda \beta$-closed set ), $f^{-1}(V)$ is $\beta$-closed in $(X, \mu)$ (denoted by $\mu \beta$-closed set).

viii) $(\mu, \lambda) \beta$-continuous if for every $\lambda$-closed set $V, f^{-1}(V)$ is $\mu \beta$-closed in $(X, \mu)$.

ix) $(\mu, \lambda) \mathrm{R}$-pre-closed if for every $\mu$-regular-closed set $U$, $f(U)$ is $\lambda$-regular-closed.

Lemma 2.5 [3]: If $\mathcal{H} \neq \phi$ is a hereditary class on $(X, \mu)$ and $f:(X, \mu) \rightarrow(Y, \lambda)$ is a function, then $f(\mathcal{H})=\{f(H): H \in \mathcal{H}\}$ is a hereditary class on $(Y, \lambda)$.

Theorem 2.6: Let $(X, \mu, \mathcal{H})$ be a hereditary generalized topological space and $(Y, \lambda)$ be a generalized topology. A function $f:(X, \mu) \rightarrow(Y, \lambda)$ is bijective, completely $(\mu, \lambda)$ irresolute and $(\mu, \lambda)$-open. If $X$ is quasi $\mu-\mathcal{H}$-regular space, then $Y$ is quasi $\lambda-f(\mathcal{H})$-regular space.

Proof: Let $y \in Y$ and $A$ be any $\lambda \pi$-closed set. Since $f$ is completely $(\mu, \lambda)$-irresolute, $f^{-1}(A)$ is $\mu \pi$-closed subset of $X$. Since $f$ is a bijection, $f(x)=y$, then $y \neq f^{-1}(x)$ for every $x \in X$. Since $(X, \mu, \mathcal{H})$ is quasi $\mu-\mathcal{H}$-regular space, there exists $\mu$-open sets $U$ and $V$ such that $x \in U$, 


\section{International Journal of Science and Research (IJSR) \\ ISSN (Online): 2319-7064 \\ Index Copernicus Value (2013): 6.14 | Impact Factor (2015): 6.391}

$f^{-1}(A)-V \in \mathcal{H}$ and $U \cap V \in \mathcal{H}$. Since $f$ is $(\mu, \lambda)$ open, $f(U)$ and $f(V)$ are $\mu$-open sets in $Y$. Also $y \in f(U), \quad$ and $\quad A-f(V) \in f(\mathcal{H}) \quad$ and $f(U) \cap f(V)=f(U \cap V) \in f(\mathcal{H})$. Hence by using lemma(2.5) $Y$ is quasi $\lambda-f(\mathcal{H})$-regular space.

Lemma 2.7 [3]: If $\mathcal{H} \neq \phi$ is a hereditary class on $(Y, \lambda)$ and $f:(X, \mu) \rightarrow(Y, \lambda)$, then $f^{-1}(\mathcal{H})=\left\{f^{-1}(H): H \in\right.$ $\mathcal{H}\}$ is a hereditary class on $(X, \mu)$.

Theorem 2.8: Let $(X, \mu)$ be a generalized topological space and $(Y, \lambda, \mathcal{H})$ be hereditary generalized topology. A function $f:(X, \mu) \rightarrow(Y, \lambda)$ is injective, Almost $(\mu, \lambda)$ closed and $(\mu, \lambda)$-continuous. If $Y$ is quasi $\lambda-\mathcal{H}$-regular space, then $X$ is quasi $\mu-f^{-1}(\mathcal{H})$-regular space.

Proof: Let $x \in X$ and $A$ be any $\mu \pi$-closed subset of $X$. Since $f$ is Almost $(\mu, \lambda)$-closed, $f(A)$ is $\lambda \pi$-closed subset of $Y$. Since $(Y, \lambda, \mathcal{H})$ is quasi $\lambda-\mathcal{H}$-regular space, there exists $\lambda$-open sets $U$ and $V$ such that $f(x) \in U$, $f(A)-V \in \mathcal{H}$ and $U \cap V \in \mathcal{H}$. Since $f$ is $(\mu, \lambda)$ continuous and injective, $f^{-1}(U)$ and $f^{-1}(V)$ are $\mu$-open sets in $X$, such that $x \in f^{-1}(U)$, $A-f^{-1}(V) \in f^{-1}(\mathcal{H}) \quad$ and $\quad f^{-1}(U) \cap$ $f^{-1}(V)=f^{-1}(U \cap V) \in f^{-1}(\mathcal{H})$. Hence by using lemma (2.7) $X$ is quasi $\mu-f^{-1}(\mathcal{H})$-regular space.

Lemma 2.9 [3]: If $\mathcal{H} \neq \phi$ is a hereditary class on $(Y, \lambda)$ and $Y$ is a subset of $X$. Then $\mathcal{H}_{Y}=\{Y \cap H: H \in \mathcal{H}\}$ is a hereditary class on $Y$.

Theorem 2.10: Let $(X, \mu, \mathcal{H})$ be a generalized topological space. If $X$ is quasi $\mu-\mathcal{H}$-regular space and $Y \subset X$ is $\mu \pi$ closed set, then $Y$ is quasi $\mu-\mathcal{H}_{Y}$-regular space.

Proof: Let $y \in Y$ and $A$ be $\mu \pi$-closed subset of $Y$ and $y \notin A$. Since $Y$ is $\mu \pi$-closed set and $Y \subset X, A$ is $\mu \pi$ closed subset of $X$. Since $X$ is quasi $\mu-\mathcal{H}$-regular space, there exist $\mu$ - open sets $U$ and $V$ such that $A-U \in \mathcal{H}$, $x \in V$ and $U \cap V \in \mathcal{H}$. If $A-U=H \in \mathcal{H}$, then $A \subset(U \cup H)$. Since $A \subset F, A \subset(F \cap(U \cup H))$ and so $A \subset(F \cap U) \cup(F \cap H)$. Therefore,

$A-(F \cap U) \subset(F \cap H) \in \mathcal{H}_{Y}, y \in(V \cap F)$. Hence $(F \cap U)$ and $(F \cap V)$ are $\mu$-open sets in $Y$ such that $A-(F \cap U) \in \mathcal{H}_{Y}, y \in V$ and
$(F \cap U) \cap(F \cap V) \in \mathcal{H}_{Y}$. Hence $Y$ is quasi $\mu-\mathcal{H}_{Y}$ regular space.

Definition 2.11: A generalized topological space $(X, \mu)$ with the hereditary class $\mathcal{H}$ is said to be Almost $\mu$ - $\mathcal{H}$ - normal space if for every pair of disjoint $\mu$-regular closed sets $A$ and $B$ there exist $\mu$-open sets $F$ and $G$ such that $A-F \in \mathcal{H}$, $A-G \in \mathcal{H}$ and $F \cap G \in \mathcal{H}$.

Theorem 2.12: Let $(X, \mu, \mathcal{H})$ be a hereditary generalized topological space and $(Y, \lambda)$ be a generalized topological space. Also let $f:(X, \mu) \rightarrow(Y, \lambda)$ is $(\mu, \lambda)$ R-pre-closed and $(\mu, \lambda)$-continuous injective function. If $Y$ is Almost $\lambda$ - $\mathcal{H}$. normal space, then $X$ is Almost $\mu-f^{-1}(\mathcal{H})$-normal space.

Proof: Let $A$ and $B$ be disjoint $\mu$-regular-closed subsets of $X$. Since $f$ is $(\mu, \lambda)$ R-pre-closed, $f(A)$ and $f(B)$ are disjoint $\lambda$-regular-closed. Since $Y$ is Almost $\lambda$ - $\mathcal{H}$-normal space, there exist $\lambda$-open sets $U$ and $V$ in $Y$ such that $f(A)-U \in \mathcal{H}, f(B)-V \in \mathcal{H}, U \cap V \in \mathcal{H}$. Then $f^{-1}(f(A))-f^{-1}(U) \in f^{-1}(\mathcal{H})$ and $f^{-1}(f(B))-f^{-1}(V) \in f^{-1}(\mathcal{H})$ which implies $A-f^{-1}(U) \in f^{-1}(\mathcal{H}), B-f^{-1}(V) \in f^{-1}(\mathcal{H})$ and $f^{-1}(U) \cap f^{-1}(V) \in f^{-1}(\mathcal{H})$. Since $\mathrm{f}$ is $(\mu, \lambda)$ continuous, $f^{-1}(U)$ and $f^{-1}(V)$ are $\mu$-open subsets of $X$. Hence $(X, \mu)$ is Almost $\mu-f^{-1}(\mathcal{H})$-normal space.

Definition 2.13: A generalized topological space $(X, \mu)$ with the hereditary class $\mathcal{H}$ is said to be quasi ultra $\mu-\mathcal{H}$ normal space if for every pair of disjoint $\mu \pi$-closed sets $A$ and $B$ there exist $\mu$-clopen sets $F$ and $G$ such that $A-F \in \mathcal{H}$, $A-G \in \mathcal{H}$ and $F \cap G \in \mathcal{H}$.

Theorem 2.14: Let $(X, \mu, \mathcal{H})$ be a hereditary generalized topological space and $(Y, \lambda)$ be a generalized topological space. Also let $f:(X, \mu) \rightarrow(Y, \lambda)$ is Almost $(\mu, \lambda) \pi$-closed and perfectly $(\mu, \lambda)$-continuous injective function. If $Y$ is Quasi $\lambda$ - $\mathcal{H}$-normal space, then $X$ is Quasi ultra $\mu$ $f^{-1}(\mathcal{H})$ - normal space.

Proof: Let $F$ and $G$ be disjoint $\mu \pi$-closed subsets of $X$. Since $f$ is Almost $(\mu, \lambda) \pi$-closed, $f(F)$ and $f(G)$ are disjoint $\lambda \pi$-closed subsets of $Y$. Since $Y$ is quasi $\lambda$ - $\mathcal{H}$ normal space, there exist $\lambda$-open sets $U$ and $V$ in $Y$ such that $f(F)-U \in \mathcal{H}, f(G)-V \in \mathcal{H}$ and $U \cap V \in \mathcal{H}$. Then $\quad F-f^{-1}(U) \in f^{-1}(\mathcal{H})$, $G-f^{-1}(V) \in f^{-1}(\mathcal{H}) \quad$ and 


\section{International Journal of Science and Research (IJSR) \\ ISSN (Online): 2319-7064 \\ Index Copernicus Value (2013): 6.14 | Impact Factor (2015): 6.391}

$f^{-1}(U) \cap f^{-1}(V) \in f^{-1}(\mathcal{H})$. Since $f$ is perfectly $(\mu$,

$\lambda$ ) -continuous, $f^{-1}(U)$ and $f^{-1}(V)$ are $\mu$-open and $\mu$ closed subsets of $(X, \mu)$. Hence $X$ is quasi ultra $\mu$ $f^{-1}(\mathcal{H})$ - normal space.

Remark 2.15: Let $(X, \mu, \mathcal{H})$ be a hereditary generalized topological space and $(Y, \lambda)$ be a generalized topological space. Also let $f:(X, \mu) \rightarrow(Y, \lambda)$ is $(\mu, \lambda) \pi$-pre-closed and $(\mu, \lambda)$-continuous injective function. If $Y$ is Quasi $\lambda-\mathcal{H}$ normal space, then $X$ is Almost $\mu-f^{-1}(\mathcal{H})$-normal space.

Proof: Let $F$ and $G$ be disjoint $\mu$-regular-closed subsets of $X$ and hence $\mu \pi$-open. Since $f$ is Almost $(\mu, \lambda) \pi$-closed, $f(F)$ and $f(G)$ are disjoint $\lambda \pi$-closed. Since $Y$ is quasi $\lambda-\mathcal{H}$ normal space, there exist $\lambda$-open sets $U$ and $V$ in $Y$ such that $f(F)-U \in \mathcal{H}, f(G)-V \in \mathcal{H}$ and $U \cap V \in \mathcal{H}$. Then

$G-f^{-1}(V) \in f^{-1}(\mathcal{H})$ $F-f^{-1}(U) \in f^{-1}(\mathcal{H})$, $f^{-1}(U) \cap f^{-1}(V) \in f^{-1}(\mathcal{H})$. Since $f$ is $(\mu, \lambda)$ continuous, $f^{-1}(U)$ and $f^{-1}(V)$ are $\mu$-open subsets of $(X, \mu)$. Hence $X$ is Almost $\mu-f^{-1}(\mathcal{H})$-normal space.

Theorem 2.16: Let $(X, \mu, \mathcal{H})$ be a hereditary generalized topological space and $(Y, \lambda)$ be a generalized topological space. Also let $f:(X, \mu) \rightarrow(Y, \lambda)$ be a bijection $(\mu, \lambda)$-Rirresolute and $(\mu, \lambda)$-open function. If $X$ is Almost $\mu$ - $\mathcal{H}$ normal space, then $Y$ is Almost $\lambda-f(\mathcal{H})$-normal space.

Proof: Proof is similar to the proof of (2.6) .

\section{Quasi $\mu_{\beta^{-}} \mathcal{H}$-regular spaces and Quasi $\mu_{\beta^{-}}$ $\mathcal{H}$-normal spaces}

Definition 3.1: A hereditary generalized topological space $(X, \mu, \mathcal{H})$ is said to be Quasi $\mu_{\beta}-\mathcal{H}$-regular space if for every $\mu \pi$-closed set $A$ and $x \notin A$, there exist $\mu \beta$-open sets $U$ and $V$ such that $A-U \in \mathcal{H}, x \in V$ and $U \cap V \in \mathcal{H}$.

Definition 3.2: Let $(X, \mu, \mathcal{H})$ be a hereditary generalized topological spaces. A space $(X, \mu, \mathcal{H})$ is said to be quasi $\mu_{\beta}-\mathcal{H}$ - normal if for every pair of $\mu \pi$-closed sets $A$ and $B$ of $X$, there exist $\mu \beta$-open sets $U$ and $V$ such that $A-U \in$ $\mathcal{H}, B-V \in \mathcal{H}$, and $U \cap V \in \mathcal{H}$.

Theorem 3.3: Let $(X, \mu, \mathcal{H})$ be a hereditary generalized topological spaces. Then the followings are equivalent:

(a) $\mathrm{X}$ is a quasi $\mu_{\beta}-\mathcal{H}$-normal space. (b) for every $\mu \pi$-closed set $F$ and $\mu \pi$-open set $G$ containing $F$, there exists a $\mu \beta$-open set $V$ such that $F-U \in \mathcal{H}$ and $c_{\mu_{\beta}}{ }^{*}(V)-G \in \mathcal{H}$.

c) For each pair of disjoint $\mu \pi$-closed sets $A$ and $B$, there exists an $\mu \beta$-open set $U$ such that $A-U \in \mathcal{H}$ and $c_{\mu_{\beta}}{ }^{*}(U) \cap B \in \mathcal{H}$.

\section{Proof:}

(a) $\Rightarrow$ (b) Let $F$ be $\mu \pi$-closed set and $G$ be a $\mu \pi$-open subset of $X$. Since $X-G$ is $\mu \pi$-closed and $F \subset G$ $F \cap(X-G)=\phi$. Since $X$ is quasi $\mu_{\beta}-\mathcal{H}$-normal space, there exist disjoint $\mu \beta$-open sets $U$ and $V$ such that $F$ $V \in \quad \mathcal{H}$ and $(X-G)-V \in \mathcal{H}$. Then $c_{\mu_{\beta}}{ }^{*}(V) \subset(X-U)$ and $(X-G) \cap{c_{\mu_{\beta}}}^{*}(V) \subset(X-G) \cap(X-U)$. Hence $c_{\mu_{\beta}}{ }^{*}(V)-G \in \mathcal{H}$.

(b) $\Rightarrow$ (c) It is obvious.

(c) $\Rightarrow$ (a) Let $A$ and $B$ be $\mu \pi$-closed sets. By (c) there exists a $\mu \beta$-open $U$ such that $A-U \in \mathcal{H}$ and $c_{\mu_{\beta}}{ }^{*}(U) \cap B \in \mathcal{H}$. Let $V=X-c_{\mu_{\beta}}{ }^{*}(U)$. Since $V$ is $\mu \beta$-open and

$V=X-c_{\mu_{\beta}}{ }^{*}(U), U \cap V \in \mathcal{H}$ Hence $X$ is quasi $\mu_{\beta^{-}} \mathcal{H}$ normal space.

Theorem 3.4: Let $(X, \mu, \mathcal{H})$ be a hereditary generalized topological space and $(Y, \lambda)$ be a generalized topological space. Also let $f:(X, \mu) \rightarrow(Y, \lambda)$ is a bijective function, completely $(\mu, \lambda)$ - continuous and $(\mu, \lambda) \beta$ - open. If $X$ is quasi $\mu_{\beta^{-}} \mathcal{H}$ - normal space, then $Y$ is quasi $\lambda_{\beta}-f(\mathcal{H})$ normal space.

Proof: Let $A$ and $B$ be $\lambda \pi$-closed subsets of $Y$. Since $f$ is completely $(\mu, \lambda)$-continuous, $f^{-1}(A)$ and $f^{-1}(B)$ are $\mu_{\pi}$ -closed subsets of $X$. Since $X$ is quasi $\mu_{\beta}-\mathcal{H}$-normal space, there exist $\mu \beta$-open sets $U$ and $V$ in $X$ such that $f^{-1}(A)-U \in \mathcal{H}, f^{-1}(B)-V \in \mathcal{H}$ and $U \cap V \in \mathcal{H}$. Since $f$ is bijective, $f\left(f^{-1}(A)\right)-f(U) \in f(\mathcal{H})$, $f\left(f^{-1}(B)\right)-f(V) \in f(\mathcal{H})$ and $f(U) \cap f(V) \in f$ $(\mathcal{H})$ and hence $A-f(U) \in f(\mathcal{H}), B-f(V) \in f(\mathcal{H})$ Since $f$ is $(\mu, \lambda) \beta$ - open, $f(U)$ and $f(V)$ are $\mu \beta$-open sets in $Y$. Hence it follows that $Y$ is quasi $\lambda_{\beta}-f(\mathcal{H})$ normal space. 


\section{International Journal of Science and Research (IJSR) \\ ISSN (Online): 2319-7064 \\ Index Copernicus Value (2013): 6.14 | Impact Factor (2015): 6.391}

Theorem 3.5: Let $(X, \mu, \mathcal{H})$ be a hereditary generalized topological space and $(Y, \lambda)$ be a generalized topological space. Also let $f:(X, \mu) \rightarrow(Y, \lambda)$ is an injective function, and Almost $(\mu, \lambda)$-closed and $(\mu, \lambda) \beta$-irresolute. If $Y$ is quasi $\lambda_{\beta}-\mathcal{H}$-normal space, then $X$ is quasi $\mu_{\beta}-f^{-1}(\mathcal{H})$ normal space.

Proof: Let $A$ and $B$ be $\mu_{\pi}$-closed subsets of $X$. Since $f$ is Almost $(\mu, \lambda)$-closed, $f(A)$ and $f(B)$ are disjoint $\lambda \pi$ closed. Since $Y$ is quasi $\lambda_{\beta}-\mathcal{H}$-normal space, there exist $\mu \beta$-open sets $U$ and $V$ in $Y$ such that $f(A)-U \in \mathcal{H}$ and $f(B)-V \in \mathcal{H}$ and $\quad U \cap V \in \quad \mathcal{H}$. Then $f^{-1}(f(A))-f^{-1}(U) \in f^{-1}(\mathcal{H})$ and $f^{-1}(f(B))-f^{-1}(V) \in f^{-1}(\mathcal{H})$ and $f^{-1}(U \cap V)=f^{-1}(U) \cap f^{-1}(V) \in f^{-1}(\mathcal{H})$ which implies $\quad A-f^{-1}(U) \in f^{-1}(\mathcal{H}) \quad$ and $B-f^{-1}(V) \in f^{-1}(\mathcal{H})$. Since $f$ is $(\mu, \lambda) \beta$-irresolute, $f^{-1}(U)$ and $f^{-1}(V)$ are $\mu \beta$-open subsets of $X$. Hence it follows that $X$ is quasi $\mu_{\beta}-f^{-1}(\mathcal{H})$-normal space.

Theorem 3.6: Let $(X, \mu, \mathcal{H})$ be a generalized topological space. If $X$ is quasi $\mu_{\beta^{-}} \mathcal{H}$-normal space, and $Y \subset X$ is $\mu \pi$ closed set, then $Y$ is quasi $\mu_{\beta}-\mathcal{H}_{Y}$-normal space.

Proof: Let $A$ and $B$ be disjoint $\mu \pi$-closed subsets of $Y$. Since $Y$ is $\mu \pi$-closed set and $Y \subset X, A$ and $B$ are $\mu \pi$-closed subsets of $X$. Since $X$ is quasi $\mu_{\beta}-\mathcal{H}$-normal space, there exist $\mu \beta$-open sets $U$ and $V$ such that $A-U \in \mathcal{H}$, $B-V \in \mathcal{H}$ and $U \cap V \in \mathcal{H}$. If $A-U=H \in \mathcal{H}$, $B-V=G \in \mathcal{H}, \quad$ then $A \subset(U \cup H) \quad$ and $B \subset(V \cup G)$. Since $A \subset Y, A \subset Y \cap(U \cup H)$ and so $A \subset(Y \cap U) \cup(Y \cap H)$. Therefore $A-(Y \cap U) \subset(Y \cap H) \in \mathcal{H}_{Y}$. Similarly $B-(Y \cap V) \subset(Y \cap G) \in \mathcal{H}_{Y}$. Hence $Y \cap U$ and $Y \cap V$ are $\mu \beta$-open sets in $Y$ such that $A-(Y \cap U) \in \mathcal{H}_{Y}$ and $B-(Y \cap V) \in \mathcal{H}_{Y}$. Hence $Y$ is quasi $\mu_{\beta}-\mathcal{H}_{Y}$-normal space.

\section{Conclusion}

In this paper we introduced new classes of spaces namely quasi $\mu-\mathcal{H}$ - regular space, quasi $\mu_{\beta}-\mathcal{H}$-regular space, quasi $\mu_{\beta^{-}} \mathcal{H}$ - normal space, Almost $\mu-\mathcal{H}$-Normal spaces, Quasi ultra $\mu$ - $\mathcal{H}$-Normal space in hereditary generalized topological spaces and derived some properties by using some basic properties of $(\mu, \lambda)$-continuity in generalized topological spaces.

\section{References}

[1] Bishwambhar Roy, On a type of generalised open sets, Applied general topology, Universidad Politecnica de Valencia.Volume 12, no. 2, pp.163-173, 2011.

[2] A.Csaszar, generalized open sets in generalized topologies., Acta Mathematica Hungaria. 106, pp.53-66, 2005.

[3] A.Csaszar, Modifications in Generalized topologies via hereditary., Acta Mathematica Hungaria. 115, pp. 29-36, 2007.

[4] R.Jamunarani and P. Jeyanthi, Regular sets in generalized topological spaces.,Acta Mathematica Hungaria, 135, pp. 342-349, 2012.

[5] K.Karuppayi, A note on RH-open sets in GTS with hereditary classes., International Journal of Mathematical Archive-5(1), pp:312-316, 2014.

[6] M.Rajamani, V.Inthumathi and R.Ramesh, A decomposition of $(\mu, \lambda)$-continuity in generalized topological spaces, Jordan J.Math.Stat.6(1),pp.15-27, 2013.

[7] J.Tong, Expansion of open sets and decomposition of continuous mappings, Rend. Circ.Mat.Polermo 43(2), 303-308, 1994. 